\title{
Prognostic impact of lymph node ratio in patients with pT1-2N1M0 non-small cell lung cancer
}

\author{
Sumin Shin ${ }^{1 \#}$, Danbee Kang ${ }^{2 \#}$, Jong Ho Cho ${ }^{1}$, Yong Soo Choi ${ }^{1}$, Jhingook Kim ${ }^{1}$, Jae Ill Zo ${ }^{1}$, \\ Young Mog Shim ${ }^{1}$, Hong Kwan Kim ${ }^{1}$
}

${ }^{1}$ Department of Thoracic and Cardiovascular Surgery, Samsung Medical Center, Sungkyunkwan University School of Medicine, Seoul, Korea; ${ }^{2}$ Department of Clinical Research Design \& Evaluation, Samsung Advanced Institute for Health Sciences \& Technology (SAIHST), Sungkyunkwan University, Seoul, Korea

Contributions: (I) Conception and design: S Shin, D Kang, HK Kim; (II) Administrative support: HK Kim; (III) Provision of study materials or patients: S Shin, HK Kim, JH Cho, YS Choi, J Kim, JI Zo, YM Shim; (IV) Collection and assembly of data: S Shin, D Kang, JH Cho; (V) Data analysis and interpretation: S Shin, D Kang, HK Kim, YM Shim; (VI) Manuscript writing: All authors; (VII) Final approval of manuscript: All authors.

\#These authors contributed equally to this work.

Correspondence to: Hong Kwan Kim, MD, PhD. Prof. Department of Thoracic and Cardiovascular Surgery, Samsung Medical Center, Sungkyunkwan University School of Medicine, 81 Irwon-ro, Gangnam-gu, Seoul 06351, Korea. Email: hkts@skku.edu.

Background: This study evaluated the lymph node ratio (LNR) defined as the ratio of the number of metastatic lymph nodes to the number of dissected lymph nodes as a prognostic factor for survival in patients with pT1-2N1M0 non-small cell lung cancer (NSCLC).

Methods: We retrospectively reviewed 413 patients with pathologic T1-2N1M0 NSCLC after complete surgical resection and mediastinal LN dissection between January 2004 and December 2012. The cutoff value for LNR was determined using $\chi^{2}$ tests, which were calculated using Cox proportional hazards regression model. Based on this model, the optimal cut-off value for LNR was 0.1.

Results: The study included 337 males and 76 females with a mean age of 62 years (range, 34-83 years). Patients with a high LNR $(\geq 0.1)$ were more likely to be female and have more adenocarcinomas compared with patients with a low LNR $(<0.1)$. The overall survival (OS) and disease-free survival (DFS) rates were significantly worse in the high LNR group than the low LNR group (OS, 55.4\% vs. 69.8\%, respectively $\mathrm{P}=0.003$; DFS, $33.2 \%$ vs. $61.7 \%, \mathrm{P}<0.001)$. In the multivariate analysis, a high $\mathrm{LNR}$ was associated with significantly worse OS [adjusted hazard ratio (aHR), 2.69; 95\% confidence interval (CI), 1.74-4.17] and DFS (aHR, 2.41; 95\% CI, 1.57-3.68).

Conclusions: LNR is an independent prognostic factor for survival in patients with pT1-2N1M0 NSCLC. These findings may provide useful prognostic information to allow the selection of patients for more aggressive postoperative therapy or follow-up strategies.

Keywords: Lung neoplasm; non-small cell lung cancer (NSCLC); lymph nodes; prognosis; risk factors

Submitted Apr 08, 2020. Accepted for publication Aug 21, 2020.

doi: $10.21037 /$ jtd-20-1611

View this article at: http://dx.doi.org/10.21037/jtd-20-1611

\section{Introduction}

Lymph node (LN) metastasis is one of the most important prognostic indicators in patients with non-small cell lung cancer (NSCLC) $(1,2)$. Therefore, adequate LN dissection is mandatory to achieve accurate nodal staging as well as to ensure metastatic LN clearance $(3,4)$. Also, adjuvant treatment based on precise staging may provide additional survival benefits $(5,6)$.

The standard treatment of NSCLC with N1 disease is pulmonary resection followed by adjuvant chemotherapy (7). The current staging system of NSCLC only adopts the 
anatomic location of $\mathrm{LN}$ metastasis $(1,2)$. However, NSCLC with the same pathologic $\mathrm{N}$ stage can exhibit heterogeneous prognoses. The 5 -year overall survival rates of N1 NSCLC range from approximately $35 \%$ to $60 \%$ according to the extent of $\mathrm{LN}$ metastases $(1,2,8)$. Considering that adjuvant chemotherapy may impart only modest benefits and while risking profound toxicity $(5,6)$, a subgroup of patients with N1 involved NSCLCs who are less likely to develop recurrence could avoid additional toxic therapies after curative surgery. Therefore, the International Association for the Study of Lung Cancer (IASLC) proposed a new $\mathrm{N}$ staging system considering the extent of LN metastasis and the N1 stage was subdivided into N1a (single metastasis) and N1b (multiple metastases), which helps to predict prognosis more accurately (8).

However, since proper assessment of the status of $\mathrm{LN}$ involvement could be affected by the quality of LN dissection as well as the extent of LN metastases (4), an additional indicator addressing both features needs to be evaluated. Lymph node ratio (LNR), which is the ratio of the number of positive LNs to the total number of LNs resected during surgery, could provide information about both the extent of LN dissection and metastasis (9). The LNR can predict survival in colon, esophageal, gastric, and pancreatic cancer patients (10-13). Some studies have investigated the prognostic significance of LNR in patients with NSCLC $(9,14,15)$, but the correlation between LNR and prognosis of N1 NSCLC was not fully investigated. Accordingly, the objective of the current study was to evaluate whether the LNR is a prognostic factor for survival in patients with pT1-2N1M0 NSCLC after complete surgical resection. We present the following article in accordance with the STROBE reporting checklist (available at http://dx.doi.org/10.21037/jtd-20-1611).

\section{Methods}

\section{Study design and population}

This was retrospective cohort study, we reviewed the medical records of all patients who underwent curativeintent surgery for NSCLC at Samsung Medical Center (a 1,961-bed referral hospital in Seoul, South Korea) between January 2004 and December 2012. During this period, 4,089 consecutive patients underwent surgical resection for NSCLC. Of these, we identified 528 patients with pathologic stage IIA-IIB/N1 NSCLC. Patients were staged according to the seventh edition of the TNM classification (1).
Patients were included in the study population if they performed complete resection with systematic mediastinal lymphadenectomy. Patients who had concomitant double primary lung cancer, who underwent limited resection (segmentectomy or wedge resection), or underwent selective LN dissection or no LN dissection were excluded. Their medical records were reviewed retrospectively to assess the clinical characteristics, pathologic results, recurrence pattern, and survival. The total numbers of metastatic LNs and resected LNs were obtained from pathologic reports, and LNRs were calculated as the ratio of the number of positive LNs to the number of resected LNs. This study was reviewed and approved by the Institutional Review Board of Samsung Medical Center (IRB no. 2018-08-188) and individual consent for this retrospective analysis was waived. The study was conducted in accordance with the Declaration of Helsinki (as revised in 2013).

\section{Preoperative staging workup}

The routine preoperative workup included pulmonary function tests, computed tomography (CT) scans of the chest and upper abdomen, ${ }^{18} \mathrm{~F}$-flurodeoxyglucose positron emission tomography (PET)/CT scans, flexible bronchoscopy, and brain magnetic resonance imaging (MRI).

\section{Operative techniques}

Operative procedures included lobectomies, bilobectomies, sleeve resections, or pneumonectomies as indicated. Systematic lymph node dissection was mandatory for all patients in the study. We avoided touching the lymph node itself and avoided rupturing the capsule of the lymph node. Mediastinal lymph node dissection consisted of en bloc resection of all nodes at stations $2 \mathrm{R}, 4 \mathrm{R}, 7,8,9$, and $10 \mathrm{R}$ for right-sided tumors and nodes at stations $4 \mathrm{~L}, 5,6$, 7, 8, 9, and 10L for left-sided tumors. When lymph node enlargement was observed or lymph node metastasis was suspected, frozen section biopsies were performed during surgery.

\section{Postoperative treatment and follow-up}

Adjuvant chemotherapy, radiotherapy, or chemoradiation was administered to patients with pathologic N1 disease provided they were able to tolerate additional treatments. Patients undergoing surgery were regularly evaluated by 
CT scan every 3 to 4 months for the first 2 years following surgery and then every 6 to 12 months thereafter. Locoregional recurrence was defined as that occurring within the ipsilateral hemithorax, including the pleura and ipsilateral mediastinal lymph nodes. Distant recurrence was defined as that developing within the contralateral hemithorax or a distant solid organ. Whenever recurrence was suspected, we attempted to obtain histological or unequivocal radiological proof. In cases lost to follow-up, a telephone interview was conducted to determine outcomes.

\section{Statistical analysis}

Descriptive statistics were used to assess patient demographic characteristics and outcomes. Continuous variables are expressed as means and standard deviations (SD). Categorical variables are expressed as numbers and proportions. Student's $t$-tests or Wilcoxon rank-sum tests were used to compare continuous variables depending on the normality of distribution, and $\chi^{2}$ tests or Fisher's exact tests were used to compare categorical variables.

The correlation between LNR and the number of positive LNs was calculated using the Pearson correlation coefficient $(r)$. The optimal cut-off value for LNR was determined using the $\chi^{2}$ score, which was calculated using a Cox proportional hazards regression model. The cut-off value was determined based on the maximum $\chi^{2}$ score and minimum $P$ value approach.

The study endpoint was overall survival (OS). Participants were included in the study at the time of surgery and were followed-up until death or the end of the study period. Disease-free survival (DFS) was defined as the time from the date of surgery to recurrence or death. Survival curves were prepared using the KaplanMeier method and were compared univariately using logrank tests. To assess the association between LNR levels and progression, hazard ratios (HR) with $95 \%$ confidence intervals (CIs) were estimated after adjusting for age, sex, stage, type of surgery (lobectomy vs. other), and adjuvant treatment. The proportional hazards assumptions were assessed using plots of the $\log (-\log )$ survival function and Schoenfeld residuals, and the analysis was split before and after 1 year since diagnosis.

In addition to categorical analyses, LNR was modeled as a continuous variable using restricted cubic splines with knots at the $5^{\text {th }}, 35^{\text {th }}, 65^{\text {th }}$, and $95^{\text {th }}$ percentiles of the sample distributions to provide a flexible estimate of the dose-response relationship between LNR and progression. The association between LNR and the hazard of progression was assessed in pre-specified subgroups defined by adenocarcinoma and others. All statistical tests were two-sided with a significance level set at 0.05 and were performed using Stata software (ver.13.0; Stata Corp., College Station, TX, USA).

\section{Results}

\section{Clinico-pathologic features}

We identified 528 patients with pathologic stage IIA-IIB/ N1 NSCLC after complete resection with systematic LN dissection from January 2004 to December 2012. We excluded patients who had concomitant double primary lung cancer $(\mathrm{n}=24)$, who underwent limited resection $(\mathrm{n}=7)$, or underwent incomplete $\mathrm{LN}$ dissection $(\mathrm{n}=84)$. A total of 413 patients met these criteria and were included for analysis (Figure 1). The patients' characteristics are summarized in Table 1. The study included 337 males and 76 females with a mean age of 61.6 years (8.8). All patients had N1 LN metastasis, and 349 patients (84.5\%) had stage IIA disease. The mean numbers of metastatic and dissected LNs were 1.8 and 26, respectively, and the mean LNR was 0.082 . The number of metastatic LNs was significantly correlated with LNR $(\mathrm{r}=0.712 ; \mathrm{P}<0.001)$ (Figure 2$)$.

\section{Optimal cut-off value for LNR}

The optimal cut-off value for LNR was 0.1 . As shown in Table 1, patients with a high LNR $(\geq 0.1)$ were more likely to be female and have more adenocarcinomas compared with patients with a low LNR $(<0.1)$. Also, the resected number of $\mathrm{LN}$ was significantly greater in the patients with a low LNR $(<0.1)$. There was no significant difference in terms of age, stage, extent of surgery, and adjuvant treatment according to LNR.

\section{Overall survival}

The median follow-up time was 41.6 months [interquartile range (IQR), 20.6-61.0 months]. During follow-up, 121 (29.3\%) patients died. Of these, 86 patients (20.8\%) died of a lung cancer-specific cause. The OS rate at 5 years was $66.6 \%$. The OS rate in patients in the high LNR group (LNR $\geq 0.1$ ) was significantly worse than that in patients with a low LNR (OS, $55.4 \%$ vs. $69.8 \%$, respectively; $\mathrm{P}=0.003$ ) (Figure 3A). 


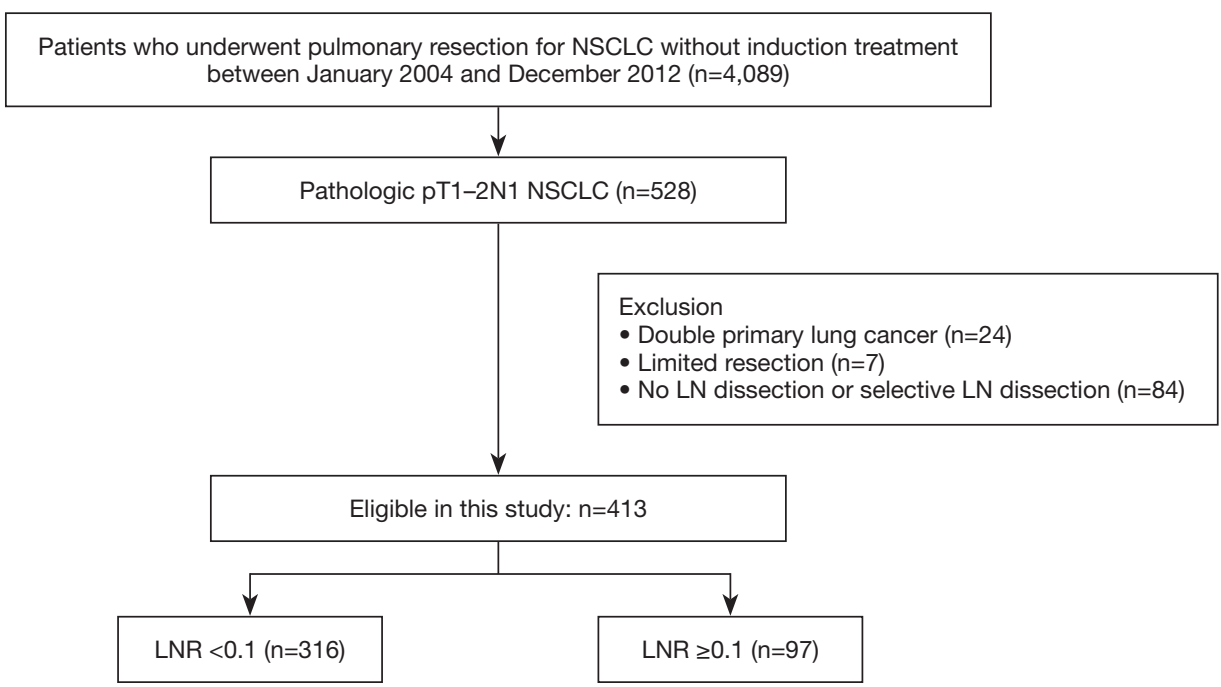

Figure 1 Flow diagram. NSCLC, non-small cell lung cancer.

Table 1 Baseline patient characteristics

\begin{tabular}{|c|c|c|c|c|}
\hline Variable & All patients $(n=413)$ & LNR $<0.1(n=316)$ & LNR $\geq 0.1(n=97)$ & $P$ value \\
\hline Sex & & & & 0.002 \\
\hline Male & $337(81.6)$ & $268(84.8)$ & 69 (71.1) & \\
\hline Female & $76(18.4)$ & $48(15.2)$ & $28(28.9)$ & \\
\hline Squamous cell & $219(53.0)$ & $188(59.5)$ & $31(32.0)$ & \\
\hline Adenocarcinoma & $159(38.5)$ & 99 (31.3) & $60(61.9)$ & \\
\hline Others & $35(8.5)$ & $29(9.2)$ & $6(6.2)$ & \\
\hline Pathologic T stage & & & & 0.271 \\
\hline pT2a & $259(62.7)$ & 206 (65.2) & $53(54.6)$ & \\
\hline pT2b & $64(15.5)$ & $47(14.9)$ & $17(17.5)$ & \\
\hline Pathologic stage & & & & 0.528 \\
\hline Stage IIA & $349(84.5)$ & 269 (85.1) & $80(82.5)$ & \\
\hline Stage IIB & $64(15.5)$ & $47(14.9)$ & $17(17.5)$ & \\
\hline No. of positive LN & $1.8 \pm 1.5$ & $1.4 \pm 0.7$ & $3.4 \pm 2.2$ & $<0.001$ \\
\hline No. of dissected LN & $26.3 \pm 11.7$ & $28.2 \pm 11.4$ & $20.2 \pm 10.7$ & $<0.001$ \\
\hline Lymph node ratio & $0.082 \pm 0.072$ & $0.053 \pm 0.022$ & $0.179 \pm 0.094$ & $<0.001$ \\
\hline
\end{tabular}

Table 1 (continued) 
Table 1 (continued)

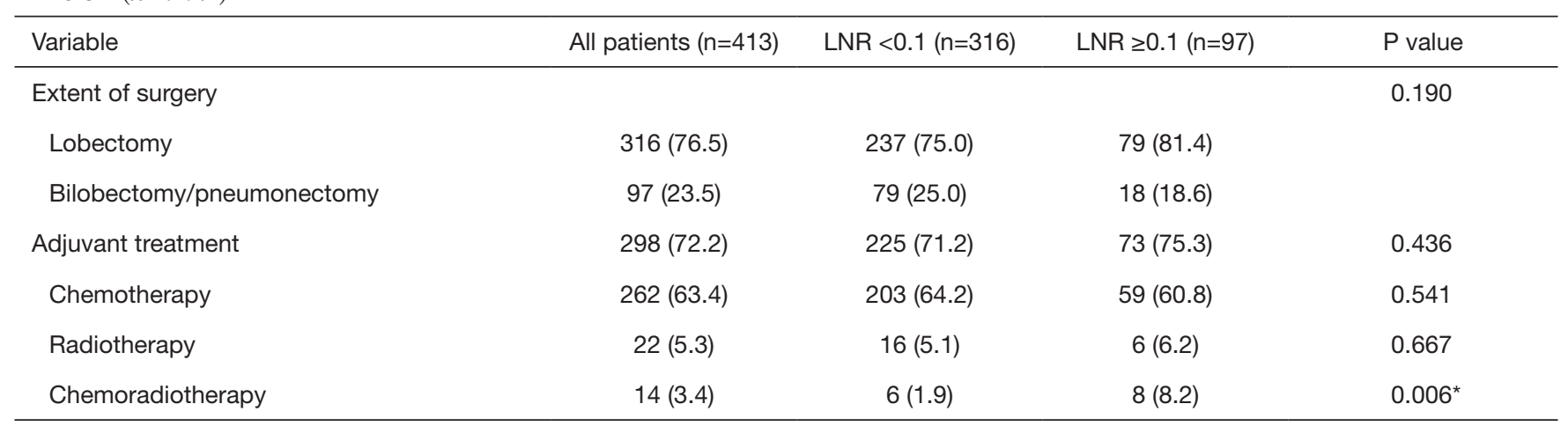

Values are number of patients (\%) or mean (standard deviation). *Fisher's exact test. LN, lymph node; LNR, lymph node ratio.

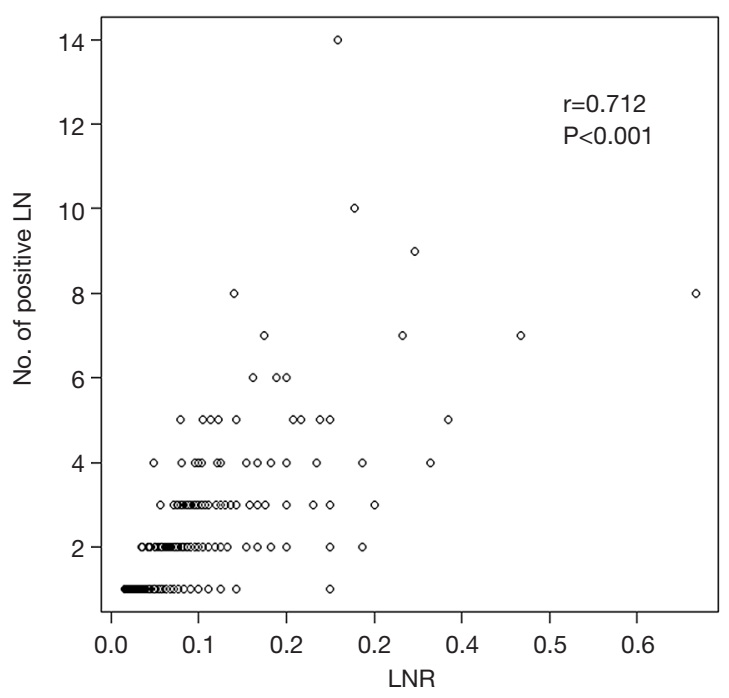

Figure 2 Correlation between metastatic lymph node and lymph node ratio.

\section{Disease free survival and pattern of recurrence}

During follow-up, 159 patients (38.5\%) developed recurrence. The pattern of recurrence was loco-regional in 45 patients $(10.9 \%)$, distant in $80(19.4 \%)$, and both in $34(8.2 \%)$. The type of recurrence was not significantly different according to LNR ( $\mathrm{P}=0.409)$. The 5 -year DFS rate in all patients was $54.9 \%$ and the 5 -year DFS rates according to LNR were $61.7 \%$ in the low LNR group (LNR $<0.1)$ and $33.2 \%$ in the high LNR group (LNR $\geq 0.1$ ). The DFS rate in the high LNR group was significantly worse than that in the low LNR group $(\mathrm{P}<0.001$; Figure $3 B)$.

\section{Prognostic impact of LNR on lung cancer-specific survival, OS, and DFS}

The HRs for OS and DFS one year after surgery according to LNR are presented in Table 2. In a multivariate analysis adjusting for clinic-pathologic variables, including age, sex, pathologic stage, histology, surgical extent, and adjuvant treatment, the high LNR group (LNR $\geq 0.1$ ) had significantly worse OS [adjusted hazard ratio (aHR), 2.69; 95\% CI, 1.74-4.17] and DFS (aHR, 2.41; 95\% CI, 1.57-3.68). The aHR for survival according to LNR are demonstrated in Figure 4. There was an increased HR for OS (Figure $4 A$ ) and DFS (Figure $4 B$ ) when patients had LNR greater than 0.1 .

The association between LNR and HR in different histologic subgroups is shown in Table 3. LNR had a greater impact on OS in adenocarcinoma (aHR, 5.02; 95\% CI, 2.56-9.82) than in other histologic subtypes (aHR, 1.68; 95\% CI, 0.90-3.15) (P value for interaction $=0.018)$.

\section{Discussion}

The present study shows that LNR was significantly associated with the prognosis of pT1-2N1M0 NSCLC after complete surgical resection in a large number of patients. Patients with a higher LNR had worse OS and DFS. In addition, the impact of LNR was robust in patients with adenocarcinoma.

Nodal metastasis is one of the most important prognostic factors in patients with NSCLC, and proper LN dissection should be mandatory to establish accurate staging and maximize the oncologic outcome $(1,2)$. The seventh TNM 

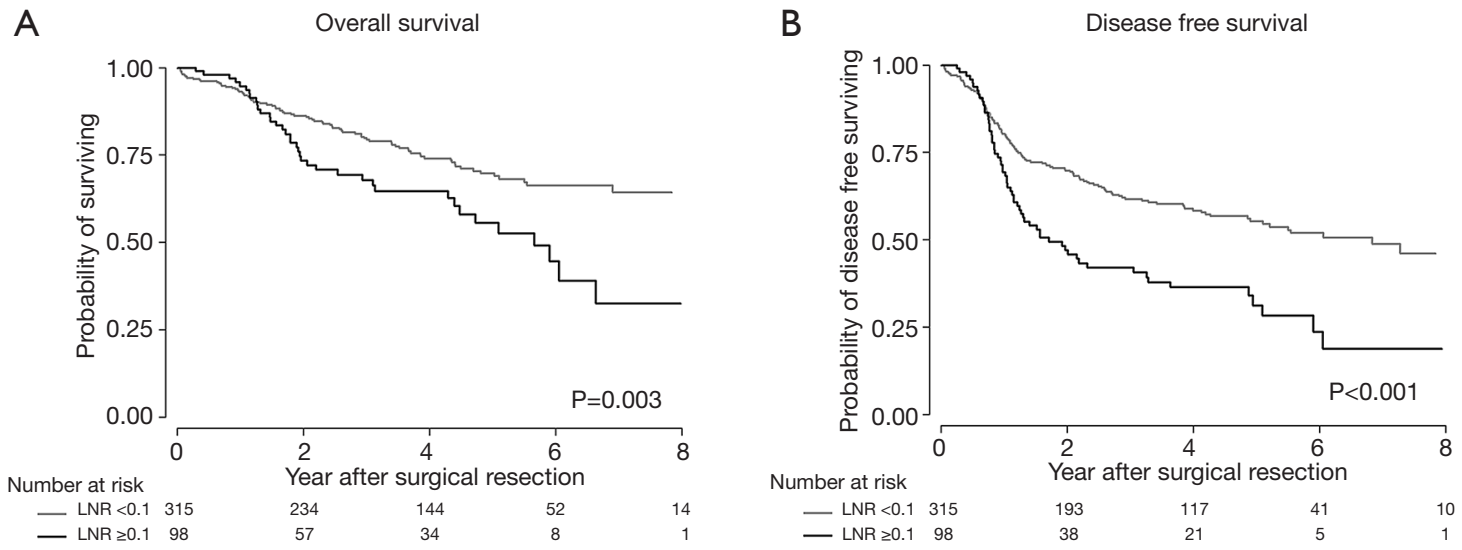

Figure 3 Overall survival and disease-free survival according to lymph node ratio (LNR). Kaplan-Meier curves showed that patients with high LNR (LNR $\geq 0.1$ ) had significantly poorer overall survival (A) and disease-free survival (B) compared to those with low LNR (LNR $<0.1$ ).

Table 2 Hazard ratio (95\% confidence intervals) for progression after 1-year associated with lymph node ratio

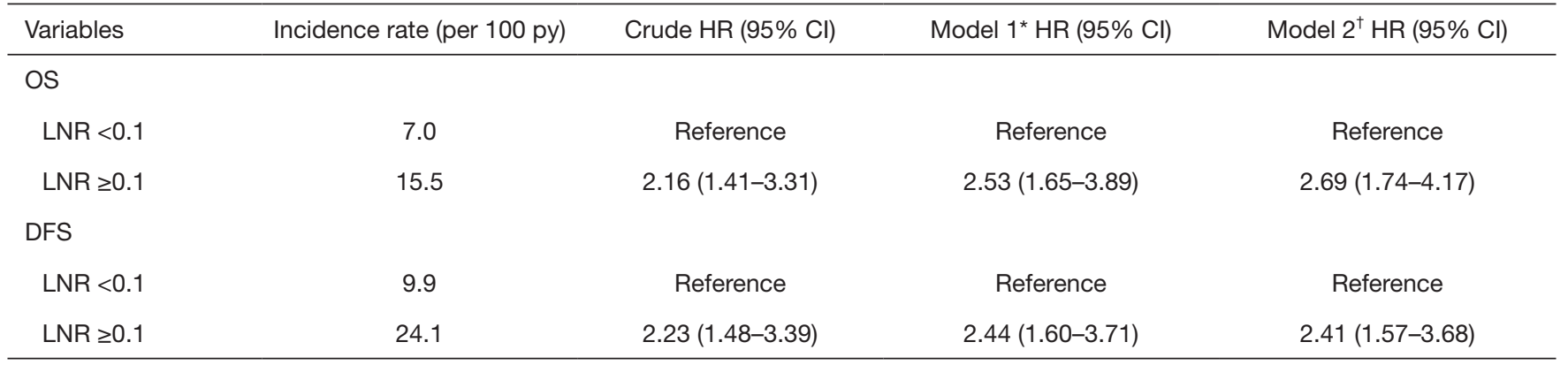

*, Model 1: Adjusted for age, sex and pathologic stage; ${ }^{\dagger}$, Model 2: Further adjusted for ADC, type of surgery (lobectomy vs. others), and adjuvant treatment. py, person-year; OS, overall survival; DFS, disease free survival; LNR, lymph node ratio; HR, hazard ratio; CI, confidence interval.
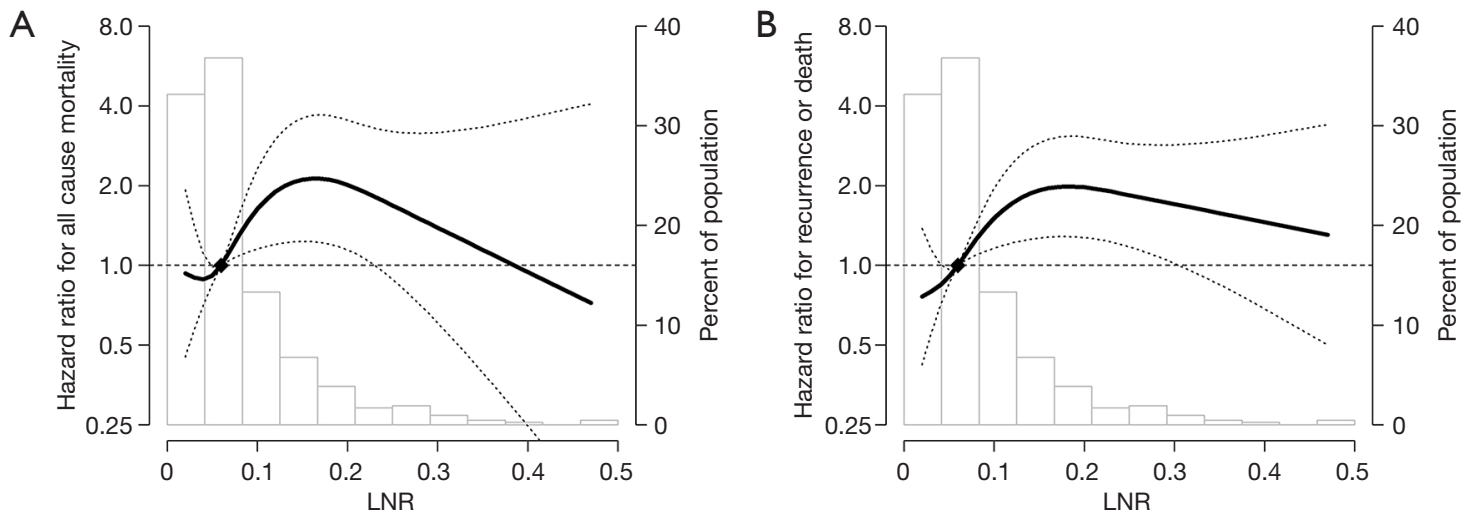

Figure 4 Hazard ratio (HR) for survival by lymph node ratio (LNR). The reference values (square dots) were set at the 50th percentile of the LNR. Y axis represents HR. HRs were obtained from Cox regression models adjusted for sex, age, pathologic stage, histology, surgical extent and adjuvant treatment. Histograms represent the frequency distributions of LNR (continuous variable). There was an increase in HR for OS (A) and DFS (B) when patients demonstrated LNR greater than 0.1. 
Table 3 Hazard ratio (95\% Confidence intervals) for progression after 1-year associated with lymph node ratio by histology

\begin{tabular}{|c|c|c|c|}
\hline Variables & ADC ${ }^{*} \mathrm{HR}(95 \% \mathrm{Cl})$ & Others* HR $(95 \% \mathrm{Cl})$ & $P$ for interaction \\
\hline LNR $<0.1$ & Reference & Reference & \\
\hline $\mathrm{LNR} \geq 0.1$ & $5.02(2.56-9.82)$ & $1.68(0.90-3.15)$ & \\
\hline Disease free survival & & & 0.065 \\
\hline $\mathrm{LNR} \geq 0.1$ & $3.32(1.96-5.61)$ & $1.49(0.73-3.03)$ & \\
\hline
\end{tabular}

*, adjusted for age, sex and stage, type of surgery (lobectomy vs. others), and adjuvant treatment. ADC, adenocarcinoma; LNR, lymph node ratio; HR, hazard ratio; $\mathrm{Cl}$, confidence interval.

staging system only defines the nodal status as N0, N1, N2, or N3 according to the anatomic location of the LN (1). The eighth TNM staging system documented that the current $\mathrm{N}$ categorization adequately predicted the prognosis of NSCLC and should be maintained, but it analyzed the association between the extent of LN involvement as a possible staging descriptor (8).

Lung cancer is the only tumor type in which nodal categorization is determined by location alone; in other tumors, the nodal status is determined by the number of LNs $(16,17)$. Several studies have suggested that the number of positive LNs has independent prognostic significance in patients with NSCLC $(14,15)$. However, the maximum number of positive LNs is limited to the number of resected LNs, and sufficient lymphadenectomy is still controversial. Samayoa et al. (3) reported an advantage in terms of OS for patients with a higher number of LNs removed; specifically, patients with nine or fewer LNs removed had a $12 \%$ increased risk of death. Similar findings were reported in other studies, and large discrepancies between the strategies of surgeons when assessing LNs was discussed $(18,19)$. This limitation can be addressed using the LNR. In the present study, the mean number of resected LNs was $>26$, suggesting that the quality of $\mathrm{LN}$ dissection was reliable. LNR correlated well with the number of positive LNs, and we believe that LNR can predict both the extent of disease and the quality of $\mathrm{LN}$ dissection.

Several studies have investigated the prognostic significance of LNR in patients with NSCLC $(9,14,15)$. There is no clear consensus regarding the cut-off value for LNR since it can be affected by the extent of disease. To clarify the impact of LNR on the prognosis of NSCLC, we confined the patient population to those with stage II disease. Previous studies included heterogeneous populations; to our knowledge, this is the first study assessing the impact of LNR on stage II/N1 NSCLC. The cut-off value in our study was 0.1 , which was smaller than those in previous studies and may be due to a discrepancy in the number of resected LNs among studies.

Based on the current cut-off value, the data indicate that a high LNR $(\geq 0.1)$ was associated with worse OS. The 5 -year OS for all patients was $67 \%$, but the 5 -year OS of patients in the high LNR group was $55 \%$. In terms of recurrence, the 5 -year DFS rate in the high LNR group was only $33 \%$. Because the Kaplan-Meier survival curves for OS and DFS crossed, we performed a Cox analysis split before and after 1 year since surgery. The HR for OS before 1 year was not significantly different according to LNR (Table S1). However, at 1 year after surgery, the risk of all-cause death and recurrence was significantly higher in patients with high LNR group compared to those with a low LNR group.

In this study, patients with a low LNR had a significantly greater number of resected LN, which may account for increased survival in patients with low LNR. Patients with a low LNR have minimal nodal involvement and may undergo better LN dissection. In other words, they can provide more accurate staging as well as more adequate nodal clearance. Meanwhile, poorer survival in patients with a high LNR could be due not only to more extensive nodal involvement but also insufficient $\mathrm{LN}$ dissection. There could be missed N2 disease in that subset. The survival of patients with a low LNR was comparable or higher than that of $\mathrm{T} 1 \mathrm{~N} 0$ or $\mathrm{T} 2 \mathrm{~N} 0$, as reported in an international database (8). Current guidelines recommend adjuvant chemotherapy to N1 LN metastasis after surgery; our data might help to select the most appropriate candidates for adjuvant treatment (7).

Interestingly, we showed that the impact of LNR was 
robust in patients with adenocarcinoma. There was a significant interaction for OS and trend for DFS according to LNR in the different histologic subgroups. The high LNR group included significantly more patients with adenocarcinoma. To clarify the effect of LNR on histology, further study with a large number of patients will be needed.

The present study has several limitations that should be discussed. First, it had a retrospective, single-center design. As it was conducted only in patients at a tertiary hospital, the results might not be generalizable to different settings. Second, the surgeon's preference regarding LN dissection can vary, which can be a cause of bias. To minimize this, we only included patients for whom four or more LN stations were evaluated. Finally, there is no clear standardized pathologic examination for LNs, number of LNs, and LNR; therefore, these parameters could vary among pathologists.

In conclusion, the LNR is an independent prognostic factor for survival in T1-2N1M0 NSCLC after complete surgical resection. These findings may provide useful prognostic information to select patients for more aggressive adjuvant therapy or follow-up strategies.

\section{Acknowledgments}

Funding: None.

\section{Footnote}

Reporting Checklist: The authors have completed the STROBE reporting checklist. Available at http://dx.doi. org/10.21037/jtd-20-1611

Data Sharing Statement: Available at http://dx.doi. org/10.21037/jtd-20-1611

Conflicts of Interest: All authors have completed the ICMJE uniform disclosure form (available at http://dx.doi. org/10.21037/jtd-20-1611). The authors have no conflicts of interest to declare.

Ethical Statement: The authors are accountable for all aspects of the work in ensuring that questions related to the accuracy or integrity of any part of the work are appropriately investigated and resolved. This study was reviewed and approved by the Institutional Review Board of Samsung Medical Center (IRB no. 2018-08-188) and individual consent for this retrospective analysis was waived. The study was conducted in accordance with the Declaration of Helsinki (as revised in 2013).

Open Access Statement: This is an Open Access article distributed in accordance with the Creative Commons Attribution-NonCommercial-NoDerivs 4.0 International License (CC BY-NC-ND 4.0), which permits the noncommercial replication and distribution of the article with the strict proviso that no changes or edits are made and the original work is properly cited (including links to both the formal publication through the relevant DOI and the license). See: https://creativecommons.org/licenses/by-nc-nd/4.0/.

\section{References}

1. Goldstraw P, Crowley J, Chansky K, et al. The IASLC Lung Cancer Staging Project: proposals for the revision of the TNM stage groupings in the forthcoming (seventh) edition of the TNM Classification of malignant tumours. J Thorac Oncol 2007;2:706-14.

2. Goldstraw P, Chansky K, Crowley J, et al. The IASLC Lung Cancer Staging Project: Proposals for Revision of the TNM Stage Groupings in the Forthcoming (Eighth) Edition of the TNM Classification for Lung Cancer. J Thorac Oncol 2016;11:39-51.

3. Samayoa AX, Pezzi TA, Pezzi CM, et al. Rationale for a Minimum Number of Lymph Nodes Removed with Non-Small Cell Lung Cancer Resection: Correlating the Number of Nodes Removed with Survival in 98,970 Patients. Ann Surg Oncol 2016;23:1005-11.

4. Liang W, He J, Shen Y, et al. Impact of Examined Lymph Node Count on Precise Staging and Long-Term Survival of Resected Non-Small-Cell Lung Cancer: A Population Study of the US SEER Database and a Chinese MultiInstitutional Registry. J Clin Oncol 2017;35:1162-70.

5. Pignon JP, Tribodet H, Scagliotti GV, et al. Lung adjuvant cisplatin evaluation: a pooled analysis by the LACE Collaborative Group. J Clin Oncol 2008;26:3552-9.

6. Arriagada R, Dunant A, Pignon JP, et al. Long-term results of the international adjuvant lung cancer trial evaluating adjuvant Cisplatin-based chemotherapy in resected lung cancer. J Clin Oncol 2010;28:35-42.

7. National Comprehensive Cancer Network. Non-Small Cell Lung Cancer 2018. Available at http://nccn.org. Accessed September 9, 2018.

8. Asamura H, Chansky K, Crowley J, et al. The International Association for the Study of Lung Cancer Lung Cancer Staging Project: Proposals for the Revision 
of the N Descriptors in the Forthcoming 8th Edition of the TNM Classification for Lung Cancer. J Thorac Oncol 2015;10:1675-84.

9. Jonnalagadda S, Arcinega J, Smith C, et al. Validation of the lymph node ratio as a prognostic factor in patients with N1 nonsmall cell lung cancer. Cancer 2011;117:4724-31.

10. Berger AC, Sigurdson ER, LeVoyer T, et al. Colon cancer survival is associated with decreasing ratio of metastatic to examined lymph nodes. J Clin Oncol 2005;23:8706-12.

11. Inoue $\mathrm{K}$, Nakane $\mathrm{Y}$, Iiyama $\mathrm{H}$, et al. The superiority of ratio-based lymph node staging in gastric carcinoma. Ann Surg Oncol 2002;9:27-34.

12. Tan Z, Ma G, Yang H, et al. Can lymph node ratio replace pn categories in the tumor-node-metastasis classification system for esophageal cancer? J Thorac Oncol 2014;9:1214-21.

13. Partelli S, Fernandez-Del Castillo C, Bassi C, et al. Invasive intraductal papillary mucinous carcinomas of the pancreas: predictors of survival and the role of lymph node ratio. Ann Surg 2010;251:477-82.

Cite this article as: Shin S, Kang D, Cho JH, Choi YS, Kim J, Zo JI, Shim YM, Kim HK. Prognostic impact of lymph node ratio in patients with pT1-2N1M0 non-small cell lung cancer. J Thorac Dis 2020;12(10):5552-5560. doi: 10.21037/jtd-20-1611
14. Wisnivesky JP, Arciniega J, Mhango G, et al. Lymph node ratio as a prognostic factor in elderly patients with pathological N1 non-small cell lung cancer. Thorax 2011;66:287-93.

15. Matsuguma H, Oki I, Nakahara R, et al. Proposal of new nodal classifications for non-small-cell lung cancer based on the number and ratio of metastatic lymph nodes. Eur J Cardiothorac Surg 2012;41:19-24.

16. UICC. TNM Classification of Malignant Tumours. 8th ed. Oxford, United Kingdom: Wiley Blackwell; 2017.

17. AJCC Cancer Staging Manual. 8th ed. New York, NY: Springer; 2017.

18. Osarogiagbon RU, Ogbata O, Yu X. Number of lymph nodes associated with maximal reduction of long-term mortality risk in pathologic node-negative non-small cell lung cancer. Ann Thorac Surg 2014;97:385-93.

19. Nwogu CE, Groman A, Fahey D, et al. Number of lymph nodes and metastatic lymph node ratio are associated with survival in lung cancer. Ann Thorac Surg 2012;93:1614-9; discussion 1619-20. 
Supplementary

Table S1 Hazard ratio (95\% confidence intervals) for progression before 1-year associated with lymph node ratio

\begin{tabular}{|c|c|c|c|c|}
\hline Variables & Incidence rate (per 100 py) & Crude HR $(95 \% \mathrm{Cl})$ & Model 1* HR (95\% Cl) & Model $2^{\dagger} \mathrm{HR}(95 \% \mathrm{Cl})$ \\
\hline LNR $<0.1$ & 7.1 & Reference & Reference & Reference \\
\hline $\mathrm{LNR} \geq 0.1$ & 5.3 & $0.75(0.28-1.99)$ & $0.80(0.30-2.12)$ & $0.87(0.32-2.31)$ \\
\hline \multicolumn{5}{|l|}{ DFS } \\
\hline LNR $\geq 0.1$ & 33.1 & 1.59 (1.02-2.48) & $1.66(1.06-2.59)$ & 1.65 (1.05-2.59) \\
\hline
\end{tabular}

${ }^{*}$ Model 1: Adjusted for age, sex and pathologic stage; ${ }^{\dagger}$ Model 2: Further adjusted for ADC, type of surgery (lobectomy vs. others), and adjuvant treatment. py, person-year; OS, overall survival; DFS, disease free survival; LNR, lymph node ratio; HR, hazard ratio; CI, confidence interval. 